\title{
Caring for Older Adults in the Radiology Department. Are You Prepared?
}

By: Beth E. Barba, PhD, RN, FAGHE; James R. Barba, MA, RT(R); and Connie Rankin, MA, RN, CHPN

Barba, B. E., Barba, J. R., \& Rankin, C. (2007). Caring for older adults in the radiology department. Are you prepared? Journal of Radiology Nursing, 26(1), 11-14.

Made available courtesy of Elsevier: http://www.elsevier.com/

*** Note: Figures may be missing from this format of the document

\begin{abstract}
:
Elders are the fastest growing segment of our population. In fact, we are in the middle of a longevity revolution. They account for $50 \%$ of hospital days and fill $60-70 \%$ of hospital beds; in addition, they make up $70 \%$ of homecare patients and $90 \%$ of nursing home residents. The numbers and percentages of older adults are continuing to rise; they are now the core business of health care, using the majority of health care services. Older adults are not the same as younger adults, and the age-related changes that are present will affect the care provided. To provide compassionate, quality care, health care professionals need to be aware of the changes associated with aging. In fact, the Joint Commission on Accreditation of Health care Organizations now requires that health care staff demonstrate age-specific competencies. This article provides an overview of normal age-related changes and strategies for caring for older adults in radiology departments, or "Clinical Alerts." (J Radiol Nurs 2007;26:11-14.)
\end{abstract}

\section{Article:}

Elders are the fastest growing segment of the population. In fact, there is a longevity revolution. In $2000,12.6 \%$ of the population was aged 65 or older. By 2030, about one in five Americans will be 65 or older, and $10 \%$ of these will be over 80 (Administration on Aging \& U.S. Department of Health and Human Services, 2004). All health care providers encounter older adults; indeed, the majority of health services are provided to older adults. They account for 50\% of hospital days and fill $60-70 \%$ of hospital beds; in addition, they make up $70 \%$ of homecare patients and $90 \%$ of nursing home residents (Healthcare in America, 2004). To provide compassionate, quality care, health care professionals need to be aware of the changes associated with aging. In fact, the Joint Commission on Accreditation of Health care Organizations (JCAHO) now requires that health care staff demonstrate age-specific competencies (JCAHO, n.d.). This article provides an overview of normal age-related changes and strategies for caring for older adults in radiology departments, or "Clinical Alerts."

\section{WHAT IS "OLD"}

Chronological age is the length of time that has passed since a person was born (The State of Aging, 2004). Aging is a universal process that is part of the life course, and there is no clearly marked point when old age be-gins. Because of Medicare and social security eligibility regulations, people tend to think of age 65 as the point of becoming "senior citizens." However, chronological age is not a good indicator of physical or psychological health (Biomarkers of Aging, 2001). Gerontologists of-ten categorize the elderly as young-old (age 65-75 years), middle-old (age 75-85 years), and old-old, or frail elderly (age 85 years and older). Although age categories can help with understanding the differences that may exist among older adults, the onset and degree of changes associated with aging vary tremendously. In addition, in the society there is so much social and cultural diversity that it is impossible to place everyone aged 65 and older into one group. This richness and diversity can be one 
of the great benefits of working with older patients. However, health care professionals cannot assume that a 60-year-old is healthier than an 80- year-old. Functional age, which is based on physical health and self-sufficiency, as well as psychosocial well-being, may be a more useful way to define age.

\section{NORMAL CHANGES ASSOCIATED WITH AGING}

Aging is not synonymous with disease; everyone ages, even in the absence of disease. It is true, however, that aging makes people more vulnerable to disease, and sometimes it is difficult to distinguish between normal aging and pathological changes. There are normal physical changes that occur over time, at different times, and to different degrees in each of the body systems. Below is a summary of these normal changes due to aging that a health care professional may see in older radiography clients.

\section{Changes in Integument}

With aging, the hair grays and the nails become thick and are easily split. The skin becomes thin and fragile because of a loss of subcutaneous fat and decreased vascularity. Skin is also drier, has fewer sweat glands, and often shows various benign skin lesions. Skin changes contribute to problems with thermal regulation, so elders are less tolerant of extremes of heat and cold (Yaar \& Gilchrest, 2001).

Clinical Alert. Be very careful in turning or lifting thin, oldest-old adults to prevent tearing skin. Use palms of hands and not fingers to lift or move an arm or leg, and roll like a log from one side to the other to achieve an oblique or lateral position. Use patient movers to assure synchronized movement of the patient from stretcher to the $\mathrm{x}$-ray table. When transferring a patient from a wheelchair, elicit the patient's cooperation and inquire how he/she moves best. It is easier for the patient if movements are initiated from the largest nearby joint (e.g., an internal movement of the hip to rotate an ankle into an internal oblique position). Make use of support devices, such as walkers and canes. Wrinkled and folded linens are irritants to the skin, so adjust them carefully. Accessories such as table pads will assure that pressure points are supported. Older patients may need blankets for warmth because x-ray rooms, tables, and image receptors are often cold.

\section{Neurological and Sensory Changes}

The brain size and weight decrease with age. Cognition is not impaired, but reaction time is slower, and elders may take longer to process information. There may be a modest decline in short-term memory, called "age-associated memory impairment" or "benign senescent forgetfulness" (Miller, 2004).

All sensory organs show some change by the age of 70. There is a higher sensory threshold and a decrease in sensory acuity. Vision changes include diminished acuity and accommodation, decreased depth perception, delayed dark to light adaptation, and increased sensitivity to glare (Cacchione, 2004). Hearing loss starts with high-frequency loss and progresses to difficulty in understanding verbal communications. Sensory changes often affect older patients' communication abilities, and they can result in misunderstanding and frustration (Wallhagen, 2002). Provide large print or audio directions, or invite family to assist with communication. The ability to taste and smell also gradually diminishes. Even the sensation of pain is altered, and the usual signs and symptoms of pain may not be present. The sense of balance may also be altered Woollacott, 2000).

Clinical Alert. Vision and hearing loss may interfere with learning or following instructions. Describe the environment and ask whether the patient can see objects. Be patient, speak clearly, and establish eye contact. Focus on the "better side;" avoid shouting; lower the pitch of the voice; and use gestures, pictures, and diagrams. Assess that patients understood the directions by asking them to repeat what was 
said. Explain that they may have difficulty hearing instructions because they may not be facing the technologist. Be aware of environmental factors such as lighting, temperature, and noise levels. Be especially careful when asking older patients to step on and off equipment because they may misjudge the distance to the floor. Be alert to signs of pain or discomfort, especially in situations when pain would be expected.

\section{Musculoskeletal System Changes}

Elders have decreased physical strength and agility because of changes in bones, muscles, and joints. Muscle cells atrophy so there is a loss of lean muscle mass. Bone density is decreased, leading to osteoporosis and an increased risk of fractures. Degenerative "wear and tear" changes occur in the joints (Miller, 2004). These musculoskeletal changes affect mobility and in-crease the risk of falls.

Clinical Alert. Take special precautions while turning or lifting older adults and having them walk. Practice fall precautions. Carefully examine the patient if there is any possibility of compression fractures, Colles' fractures of the wrist, and hip fractures. Be aware of exposure factors, and use combinations that will minimize voluntary and involuntary motion. Consider changes in the absorption coefficient of parts due to aging changes and pathological changes. Be prepared to accommodate alterations to positioning criteria through alternate positions of the $\mathrm{x}$-ray tube and image receptor when the patient is unable to move into or maintain a particular position. Know your facility's fall prevention and restraint policies.

\section{Cardiovascular Changes}

As people age, blood vessels become less elastic and the wall of the heart thickens. Cardiac reserve is diminished, leading to decreased performance under stress. Changes include a tendency toward orthostatic hypo-tension and a gradual increase in systolic blood pressure. The peripheral circulation decreases, especially in the feet, increasing the risk of injury to the lower extremities. Cardiovascular changes can cause dizziness, syncope, and fatigue (Lakatta, 2000).

Clinical Alert. Observe clients for decreased endurance. Be specific when assessing cardiac status by asking questions such as, "Are you tired?," "Are you comfortable?," "Do you feel faint?," and "Can you continue with more x-ray images?" Dizziness and syncope contribute to the risk of falling. Have the client sit up for a minute or so before standing to allow blood pressure to stabilize. Be especially careful to avoid in-jury to the lower extremities when positioning patients.

\section{Respiratory Changes}

Aging lungs become smaller, and bony changes in the thorax reduce lung distention. This leads to decreased lung capacity and a decline in the diffusing capacity needed for gas exchange. Medications can cause slow, shallow breathing. Age-related changes in the respiratory system are subtle and gradual. Healthy older adults can usually compensate for these changes. However, when stressors such as illness and anesthesia place extra demands on the body, changes in overall function of the respiratory system may be observed (Miller, 2004).

Clinical Alert. Observe for shortness of breath, especially with exertion. Observe the patient's respiratory rate and effort, skin color, and pulse rate. Place older patients in an upright position when they show respiratory distress. When taking chest $\mathrm{x}$-rays, tell patients to take two deep breaths and hold the third to get the deepest inspiration. Activity tolerance may be limited, so provide rest periods. 


\section{Gastrointestinal Changes}

Teeth may be worn or missing, making chewing food difficult. Appetite may be affected by decreased taste and smell, and slower gastric emptying can produce early satiety. The entire gastrointestinal tract slows. Constipation is a common problem because of slowed peristalsis, reduced abdominal muscle strength, inadequate exercise, and reduced food and fluid intake (Prather, 2000).

Clinical Alert. There is increased risk of stool impaction and bowel obstruction. When performing imaging examinations in the abdominal region, ask the patient about bowel habits and last movement. Be especially diligent with postexamination instructions for barium and other contrast studies because of the probability of constipation.

\section{Renal/Genitourinary Changes}

Kidney mass decreases, and there is often arteriosclerosis of renal arteries. Function of the glomerular filtration system declines, causing less efficient dilution or concentration of the urine, and imprecise serum creatinine values. The bladder loses tone and the ability to expand, and pelvic floor muscles and the urinary sphincter weaken (Miller, 2004). Many male elders have benign prostatic hypertrophy. These changes can lead to stress incontinence or retention, frequency, and nocturia (Newman \& Palmer, 2003).

Clinical Alert. Before administering contrast agents, realize that creatinine levels should not be considered independently, provide adequate hydration, and note nephrotoxic drugs (Bettmann, 2004). Older adults may not have the capacity to hold urine for an extended time. Inquire about the need to urinate before beginning the x-ray examination, explaining the approximate length of the procedure. If they ask to urinate, assist them to the toilet as quickly as possible. Be sure there are no obstructions between the patient and the bathroom and have supplies readily available. Provide a commode or bedpan/urinal if the toilet is too far away. Because older adults are more prone to dehydration, be alert for symptoms such as confusion, lethargy, or dry tongue and mucous membranes.

\section{Endocrine System Changes}

The very old have a delayed or inadequate response of the immune system to infectious agents. When infections are contracted, they are more likely to be fatal than they would be in a younger adult. Because of adrenal changes, older adults have a diminished ability to respond to physiological stressors.

Clinical Alert. Abide by universal precautions. Always wash your hands between patients and assist older patients with hand washing, when appropriate. Keep equipment clean. Adjust the environment to reduce both physical and psychological stressors as much as possible.

\section{Psychosocial Changes}

Older patients face numerous psychosocial challenges. Loss of significant relationships through death of a spouse, friends, or family members is common. Many elders also must adjust to retirement, which may mean reduced income. Loss of a sense of control over decision-making often accompanies loss of health because of the attitudes of family and caregivers (Barba, Tesh, \& DeBrew, 2005). Often relocation from the family home is necessary because declining health leads to functional limitations. Personality does not change as people age, although some aspects of personality may appear exaggerated (Miller, 2004). The ability to cope is influenced by individual coping styles and maturity level. Social support, financial assets, social status, self-esteem, and religious beliefs also influence how the elderly deal with stressful life events (Antonucci, 2001). Depression is the most common psychiatric 
problem, and substance abuse and anxiety disorders are also seen in the elderly population (Hall, Hall, $\&$ Chapman, 2003). Overall, however, older patients are likely to accommodate to the situation at hand, and they are usually accepting of the authority of health care professionals (Miller, 2004).

Clinical Alert. Be sensitive to older patients' fears and anxieties about loss of health and functional abilities. Explain all procedures carefully and patiently. Understand that instructions may need to be repeated because anxiety combined with sensory changes can influence older patients' comprehension. Encourage family caregivers to be present, if appropriate.

\section{SUMMARY}

It is important to recognize that older adults are a diverse group of people who experience some common changes as they age. Older adults are not the same as younger adults, and the age-related changes that are present will affect the care provided. The numbers and percentages of older adults are continuing to rise; they are now the core business of health care, using the majority of health care services. Being aware of age-related changes will help health care professionals provide sensitive and effective age-appropriate care.

\section{References}

Administration on Aging., \& U.S. Department of Health and Human Services. (2004). Statistics, Retrieved from, http:// www.aoa.dhhs.gov/prof/Statistics/statistics.asp, May 1, 2006.

Antonucci, T.C. (2001). Social relations: An examination of social networks, social support, and sense of control. Birren, J.E.Schaie, K.W. Handbook of the psychology of aging (5th ed.). San Diego, CA: Academic Press, pp. 427-453.

Barba, B.E., Tesh, A.S., \& DeBrew, J. (2005). The hospitalized older adult: A teaching manual for geriatric nursing care. Greensboro, NC: The University of North Carolina at Greensboro.

Bettmann, M.A. (2004). Frequently asked questions: Iodinated contrast agents. Radiographics, 24(Suppl. 1), S3-S10.

Biomarkers of aging: From primitive organisms to man. (2001) New York, NY: International Longevity Center.

Cacchione, P.Z. (2004). Nursing interventions for older adults with visual or auditory sensory impairments. Healthy Aging, 11, 7-8.

Hall, R., Hall, R., \& Chapman, M. (2003). Identifying geriatric patients at risk for suicide and depression. Clinical Geriatrics, 10(11), 36-44.

Health care in America, trends in utilization. (2004) (DHHS Publication no. 2004-1031:03-0357). Hyattsville, MD: National Center for Health Statistics.

Joint Commission on Accreditation of Health Care Organizations. (n.d.). JCAHO accreditation pop manual. Retrieved from www.popmanual.com/index.cfm, May 1, 2006.

Lakatta, E.G. (2000). Cardiovascular aging in health. Clinics in Geriatric Medicine, 16(3), 419-444. Miller, C. (2004). Nursing for wellness in older adults (4th ed.). Philadelphia, PA: Lippincott Williams \& Wilkins.

Newman, K.K., Palmar, M.H. (2003). State of the science on urinary incontinence. American Journal of Nursing, 103(Suppl.), 54-56.

Prather, C.M. (2000). Constipation, diarrhea, and fecal incontinence. Beers, M.H. Berkow, R. The Merck manual of geriatrics (3rd ed.). Washington, DC: Merck Institute of Aging \& Health.

The state of aging and health in America 2004. (2004) Washing-ton, DC: Merck Institute of Aging \& Health.

Wallhagen, M.T. (2002). Hearing impairment. Annual Review of Nursing Research, 20, 341-368. 
Woollacott, M.H. (2000). Systems contributing to balance disorders in older adults. The Journals of Gerontology, 55A, M424-M438.

Yaar, M., Gilchrest, B.A., Skin aging: Postulated mechanisms and consequent changes in structure and function. Clinics in Geriatric Medicine, 17(4), 617-630. 